\title{
Cybercriminalité ou "Broutage" et Crimes Rituels à Abidjan: Logiques des Acteurs et Réponses au Phénomène Cas des Communes de Yopougon et d'Abobo
}

\author{
Raymond Nébi Bazare \\ Docteur en Criminologie, Sociocriminologue, Maître Assistant \\ Université Félix HOUPHOUET Boigny de Cocody-Abidjan, Côte d'Ivoire \\ Bamba Ladji \\ Docteur en Criminologie, Maître Assistant, UFR Criminologie \\ Université Félix HOUPHOUET Boigny de Cocody-Abidjan, Côte d'Ivoire \\ Dolle Kadidja \\ Doctorant à l'UFR Criminologie \\ Université Félix HOUPHOUET Boigny de Cocody-Abidjan, Côte d'Ivoire \\ doi: 10.19044/esj.2017.v13n23p104 URL:http://dx.doi.org/10.19044/esj.2017.v13n23p104
}

\begin{abstract}
The aim of this study is to show the existence of a link between ritual crimes and the cybercrime that takes place in the territory of Abidjan. It shows the logics of the actors and proposes effective policies to combat this phenomenon. It was carried out in two municipalities of Abidjan, namely: Yopougon (with the largest demographic plan) and Abobo. The survey was conducted with 50 people, including 20 in Yopougon and 30 in Abobo. The survey was conducted on the basis of reasoned choice using data collection techniques such as documentary study, observation, and inquiry. The data were analyzed both quantitatively and qualitatively. The results show that there is no particular type of cybercriminals who commit ritual crimes. Everyone does it, but for different purposes. Most of them spell the target in order to annihilate anyone that is resistance and is refusing. For others, mystical rituals help them in their understanding of how to escape the police and justice. They involve other actors in the network on their activities. In this case, the agents of western union, targets, security agents and others are also involved.
\end{abstract}

Keywords: Magic of street, Crime, Abnormality, game of the roles, the victimization 


\section{Résumé}

Cette étude a pour objectif de montrer l'existence de lien entre les crimes rituels et la cybercriminalité qui a court sur le territoire d'Abidjan. Elle vise à appréhender les logiques des acteurs et à proposer des politiques de lutte efficaces contre ce phénomène. Elle a été menée dans deux communes d'Abidjan, à savoir Yopougon (au plan démographique la plus importante) et Abobo qui la seconde. L'enquête a été menée auprès de 50 personnes dont 20 à Yopougon et 30 à Abobo, sur la base du choix raisonné ; et à l'aide des techniques de recueil des données que sont l'étude documentaire, l'observation et l'enquête interrogation. Les données ont été analysées aussi bien quantitativement que qualitativement. Il en ressort qu'il n'y a pas un type particulier de cybercriminels qui commettent les crimes rituels. Tous s'y adonnent, mais à des fins différentes : la plupart pour envoûter la cible afin d'annihiler toute volonté de résistance et de refus de la cible potentielle. Pour d'autres, les rituels mystiques les aident dans leur entendement, à échapper à la police et à la justice. Ils envoûtent d'autres acteurs du réseau afin de les impliquer tous dans leurs activités. En l'occurrence ce sont les agents de western union, cibles, agents de sécurité et autres qui se voient impliquer.

Mots-clés: Magie de rue, Délinquance, Déviance, jeu des rôles, victimisation

\section{Introduction}

\section{Quelques repères théoriques}

En Côte d'Ivoire et plus particulièrement à Abidjan, le taux de pénétration de l'outil informatique est de $70.58 \%$ en 2010 (ATCI, 2010). Cette situation a favorisé l'augmentation du taux de chômage de 6,2\% en 2002 à 15,7\% en 2008, puis en 2012 avec un taux de 9,4\% (INS, 2008; AGEPE, 2012). Les cyber- délinquants ont comme alternatives à leur oisiveté et manque d'emploi, l'arnaque sur le web, malgré leur connaissance des risques encourus (arrestation par la plateforme de lutte contre la cybercriminalité [PLCC] et emprisonnement), au détriment des études et de la formation. Ainsi, à côté de la criminalité violente traditionnelle, se manifeste une nouvelle forme de criminalité plus astucieuse communément appelée « broutage 3G » dans le jargon ivoirien. : Une activité qui depuis un moment ne prospère plus, du fait que les victimes sont devenues prudentes et les luttes donnent des résultats tangibles. Alors ces jeunes vont associer à la cybercriminalité des rites mystiques qui peuvent aboutir à des crimes rituels. Et ceci dans le but d'agir sur la volonté des potentielles victimes en vue d'un enrichissement rapide. Inventer dans les années 90, le terme cybercriminalité a été employé par un sous-groupe des pays du G8 pour la première fois pour désigner l'ensemble des délits commis via les réseaux internet. Ce 
phénomène a non seulement, pris une proportion importante, mais s'est revêtue de formes très particulières en Côte d'Ivoire et surtout à Abidjan. D’aucuns parlent d'utilisation de pouvoirs mystiques appelés «ZAMOU » dans le jargon cybercriminel ivoirien, qui consiste à s'attacher les services d'un mystique-marabout ou féticheur afin de faciliter la réalisation et assurer le succès des arnaques sur internet. En termes ivoiriens, il s'agit, pour les cybercriminels, «d'attacher » (envoûter) la victime, afin que celle-ci cède le plus facilement aux mensonges mis en jeu, et d'impliquer plusieurs autres acteurs pour soutirer le maximum d'argent aux victimes. Dans la pratique, les actes du «zamou » peuvent être commis à des fins de trafics d'organes, politiques (campagnes électorales, nominations, remaniements ministériels, promotion...), d'impunité (échapper aux mailles de la justice), d'arnaques, broutage ou cybercriminalité (envoûter la cible afin de la contraindre à remettre la somme demandée). Toutefois, ils répondraient au même principe à savoir : pactiser avec une entité mystique en offrant un sacrifice extrême (sang humain, partie du corps, secrétions humaines, etc....). C'est ce phénomène qui nous intéresse dans cette étude : les crimes rituels rattachés à la cybercriminalité ou au «broutage».

Quand nous parcourons la littérature scientifique sur la question, nous constatons que les termes «crimes rituels » et « cybercriminalité », mis en rapport, ont très peu intéressé les chercheurs. Et pourtant ce phénomène s'intensifie et prend de nouvelles formes au fur et à mesure que les années passent. Pour preuve, Bamba et Bazare (2016) se sont contentés d'expliquer le mécanisme de catégorisation sociale qui régit l'univers des cybercriminels, en mettant l'accent sur les pratiques festives du brouteur (sexe, alcoolisme, psychotropes, etc.). Ainsi, bien qu'ayant pu mettre en lumière la complexité $\mathrm{du}$ processus de typologisation sociale du cybercriminel ivoirien et des pratiques festives qui s'y rattachent (notamment le caractère ritualiste des pratiques sociales existantes), ils n'ont pu aborder la question du croisement entre les deux crimes que sont la cybercriminalité et les crimes rituels. Vladimir (2013), désigné par de nombreux spécialistes comme l'un des pôles les plus actifs des études sur la cyberescroquerie au niveau de l'Afrique de l'Ouest, a traité la question des pratiques occultes voir sataniques en relation avec la cybercriminalité. Et ce, autour de deux axes : le premier fait allusion aux mécanismes qui servent à faciliter la réalisation et assurer la pérennité des arnaques sur internet afin que les cibles cèdent le plus facilement aux mensonges et stratagèmes mis en œuvre par les cyberescrocs. Dans le second axe, il s'agit, pour les cybercriminels ou «brouteurs» dans le jargon ivoirien, de pactiser avec une entité mystique (marabout, féticheur, génie, etc.) en offrant un sacrifice extrême (sang humain, partie de son corps, secrétions humaines, etc....) dans le but de connaitre le succès dans leurs entreprises criminelles. Toutefois Vladimir n'a pas pu relever les facteurs 
explicatifs de ces crimes rituels rattachés à la cybercriminalité. Soudan (2014) quant à lui, aborde l'expansion de la pratique des crimes rituels dans les pays africains. Pour lui, ces pratiques ont donné naissance à un trafic d'organe humain en Afrique. À sa suite, Tsimi et Rifoe (2015) affirment que l'immigration clandestine des ressortissants gabonais favorise dans la capitale camerounaise la pratique des crimes rituels qui s'impose comme un véritable passeport CEMAC. Autour de deux axes, ils présentent cette pratique : dans le premier, ils parlent du mode opératoire des crimes rituels et des conséquences engendrés par ces actes. Dans le second axe, ils abordent la question de corruption des agents de l'État face à ces pratiques rituelles; ce qu'ils dénoncent en ces termes:»' les prélèvements seraient ensuite fournis à des commanditaires dont on imagine qu'ils ont le bras très long puisqu'ils ne sont jamais dénoncés, en tout cas, pas publiquement »'. Si Soudan, Tsimi et Rifoe ont eu le mérite de décrire le phénomène des crimes rituels et de relever leurs conséquences, ils n'ont pu pousser leurs investigations loin afin de nous éclairer sur le rapport de ces actes avec la cybercriminalité. Vallet (2005), de son côté, aborde le problème de la réglementation des contenus illicites circulants sur les réseaux sociaux. Exposant les dommages graves à savoir les pertes financières et la destruction de l'outil informatique que les contenus illicites émis par les cybercriminels provoquent, l'auteur propose comme solution la censure de ces contenus illicites. Proposition que nous jugeons insuffisante parce que ne prenant pas en compte d'une part les voies à suivre pour assurer une bonne réglementation de ces contenus qui circulent sur le net, et d'autre part la question des crimes rituels dans la cybercriminalité. Le Cabinet américain «IOVATION», spécialisé dans la protection des entreprises en ligne, soulignait dans son dernier rapport de 2012 que : «les cybercriminels ghanéens opéraient entre minuit et 1 heure du matin ». Dans cette étude, l'accent est mis sur les modes opératoires des cybercriminels ou «sakawaboys » et la corruption qui existe entre les cybercriminels, les autorités policières et les agents de transfert de fonds. Le rapport nous laisse perplexes quant à une éventuelle esquisse de solutions pour une lutte plus efficace contre le phénomène. Jeune Afrique (2015) mentionne le désintérêt des jeunes pour les études et la multiplication de pratiques mystiques au profit de la cybercriminalité. Si à la différence des autres publications, Jeune Afrique a eu le mérite d'aborder le rapprochement des crimes rituels à la cybercriminalité, aucune suggestion n'est faite pour les circonscrire. Au terme de l'examen de tous ces travaux, relatifs à une jeunesse ou une population africaine prédatrice appelée "brouteur» ou cybercriminel, on note que la psychose autour des crimes rituels a pris une ampleur sans précédent. Ce phénomène favorise une appétence au surnaturel, à l'inconnu, au mystère et invite à rebâtir le pacte social. C'est d'ailleurs la raison pour 
laquelle notre étude s'intéresse à la problématique de la réalité sociale des crimes rituels et de leur association à la cybercriminalité. Elle brandit un éventail de l'insécurité qui se manifeste aux yeux de tous, mais qui paradoxalement demeure inconnu à cause des implications multiples de toutes les catégories socioprofessionnelles. Convient — il de les analyser sous l'angle de déterminants socio — politiques ou d'insécurité réelle ? Estce qu'il ne s'agit pas d'homicides «normaux » d'hommes et de femmes envieuses, jalouses, sadiques, sanguinaires, qui déguisent leurs actes en crimes rituels, histoire de détourner l'attention des acteurs de la sécurité ? Peut-être que ces mutilations criminelles n'ont aucun lien avec l'octroi de pouvoirs mystiques. Si c'est le cas, les «' brouteurs »' seraient-ils simplement entre deux crimes : la cybercriminalité et le trafic d'organes ? À quoi servent donc les crimes rituels dans «' la pratique du broutage »' ou de la cybercriminalité ? Comment les brouteurs ou cybercriminels arrivent-ils à commettre ces crimes rituels ? À quel taux ce phénomène est-il ancré dans le quotidien des Abidjanais?

Notre objectif consiste à décrire les manifestations des crimes rituels liés à la cybercriminalité, identifier les facteurs explicatifs de ces crimes rituels, relever les conséquences de ce phénomène et proposer des politiques de lutte efficaces contre cette réalité sociale.

Pour atteindre ces objectifs et répondre à nos questions de recherches, nous formulons les hypothèses suivantes:

H1 : Les «brouteurs » commettent des crimes rituels en vue d'envoûter la cible pour lui soutirer facilement de fortes sommes d'argent.

$\mathrm{H} 2$ : Les crimes rituels sont commis dans le cadre du «broutage » en vue de favoriser les conditions mystiques susceptibles de permettre au brouteur d'opérer en toute impunité.

H3 : Les crimes rituels associés au «broutage » constituent pour les « brouteurs » un moyen d'occulter leur activité de trafic d'organe.

\section{Méthodologie}

\section{Terrain d'étude}

Les résultats du RGPH 2014 indiquent que la ville d'Abidjan comprend 4.707.000 habitants, soit $20 \%$ de la population ivoirienne. La commune de Yopougon est la plus peuplée avec 1.071 .000 personnes. Yopougon a une Densité de 8984 hab. $/ \mathrm{km} 2$ et une Superficie 15306 ha = $153,06 \mathrm{~km} 2$. Yopougon est en premier notre lieu de résidence. Deuxièmement, la plupart des institutions y sont représentées (trésor, impôt, cyber café, établissements hospitaliers et scolaires, etc.). Troisièmement, la commune est connue pour son ambiance festive dans les bars et maquis où se déroule constamment «le concept du "travaillement" et du boucan ». La seconde commune choisie est Abobo. Elle est située au Nord d'Abidjan. 
C'est la seconde commune la plus peuplée d'Abidjan après celle de Yopougon avec une population estimée environ 1.030.658 habitant d'après les statistiques de l'Institut National de la Statistique (1'INS, 2014) et d'une superficie de 10000 hectares pour soit une densité de 167 habitants à 1'hectare. Comme Yopougon, la commune d'Abobo compte de nombreux quartiers dont 19 quartiers précaires pour Abobo (Colatier, Sagbé 1 et 2, l'île verte, Kennedy, Agnissankoi, Bocabo et Abobo Nany pour ne citer que ceuxlà. Dans ces aires, la criminalité est bien réelle. Et à ce propos, nous pouvons citer le cas d'un agent de sécurité privée posté devant le lycée moderne d'Abobo qui a été décapité dans le mois d'octobre 2015. Ce sont ce type de faits qui justifient encore l'importance de notre étude dans ces communes.

\section{Échantillon}

Dans l'impossibilité de recueillir les réponses de toute une population (population mère), nous nous sommes référés à un échantillon de personnes récoltées à Yopougon (20 personnes) et à Abobo (30 personnes). À l'aide de la méthode des quotas, nous avons pu obtenir un échantillon.

Ainsi nous avons interrogé 50 personnes dont 20 à Yopougon et 30 à Abobo qui se répartissent comme suit :

- $\quad 10$ ex-cybercriminels professionnels qui pratiquaient les crimes rituels ; (5 à Yopougon, 5 à Abobo)

- $\quad 02$ féticheurs en relation avec des cybercriminels (1 à Yopougon, 1 autre à Abobo)

- $\quad 14$ familles de victimes ; (5 à Yopougon, 9 à Abobo)

- $\quad 15$ gérants de cybercafés ; $(4,11$ à Abobo)

- $\quad 04$ forces de l'ordre ; (2 à Yopougon, 2 à Abobo)

- 5 professionnels des NTIC. (2 à Yopougon, 3 à Abobo)

Tableau 1. Tableau récapitulatif de la constitution de notre échantillon concernant certaine population enquêtée à Yopougon et à Abobo

\begin{tabular}{|c|c|}
\hline Population cible & Échantillon \\
\hline Chefs coutumiers & 30 dont 15 à Yopougon et 15 à Abobo \\
\hline $\begin{array}{c}\text { Autorités politiques et } \\
\text { administratives }\end{array}$ & 30 dont 15 à Yopougon et 15 à Abobo \\
\hline Parents de victimes & 15 dont 7 à Yopougon et 8 à Abobo \\
\hline Auteurs de délits & 10 dont 4 à Yopougon et 6 à Abobo \\
\hline Étudiants et élèves & 60 dont 30 à Yopougon et 30 à Abobo \\
\hline Total & \multicolumn{1}{c}{145} \\
\hline
\end{tabular}

Source_: Enquêtes 


\section{Méthodes de recherches et techniques de recueil des données Méthodes de recherches \\ Méthode Systémique}

Selon Elkaim (1995), la méthode systémique se distingue des autres méthodes par sa façon de comprendre les relations humaines. Elle nous a permis de comprendre que l'individu est influencé à la fois par ses intentions, celles des autres, et celles des possibilités du milieu et/ou du système.

\section{Méthode Clinique}

Pour Douville (2006), la méthode clinique est une démarche scientifique de recherche et de production de savoir. Ainsi, à l'aide d'un questionnaire nous avons été au cheveu des cybercriminels.

\section{Techniques de recueil}

Cette étude a nécessité l'utilisation de trois (3) techniques de collecte de données que sont: l'étude documentaire, par observation puis le questionnaire et l'entretien

\section{Étude Documentaire}

Cette étude a été importante dans les investigations, car elle a contribué à l'examen d'une gamme variée de documents (documents scientifiques, revues...) qui ont traité cette forme de crimes en relation avec la cybercriminalité.

\section{Observation}

L'étude par observation nous a permis d'observer, d'expliquer l'environnement dans lequel le phénomène est pratiqué et de déterminer les conditions socio-économiques des cybercriminels. Ensuite, énumérer les obstacles que rencontrent les forces de l'ordre et de sécurité dans l'exercice de leurs fonctions.

\section{Enquête Interrogation Entretien}

Nous avons utilisé l'entretien semi-directif. Ainsi à l'aide de guide d'entretien composé de questions semi-ouvertes nous avons pu donner la possibilité à nos interlocuteurs de s'exprimer.

\section{Questionnaire}

Le questionnaire que nous avons utilisé s'est fait en deux catégories. Les populations enquêtées se sont exprimées en toute liberté et dans le sens 
précis de la recherche. Notons que nous avons utilisé deux types de questionnaires constitués de questions ouvertes et fermées.

\section{Méthodes d'analyse}

$\mathrm{Au}$ cours de cette recherche deux méthodes d'analyses de données : la méthode d'analyse qualitative et la méthode d'analyse quantitative.

\section{Analyse Qualitative}

Concernant la méthode d'analyse qualitative l'on doit noter qu'elles ont permis d'expliquer les réactions, les opinions des populations enquêtées... sur ce phénomène.

\section{Analyse Quantitative}

Cette méthode d'analyse quantitative permet d'établir les différentes formes des crimes opérés par les cybercriminels et de les classer par type.

\section{Résultats}

\section{Manifestations des crimes rituels associées à la cybercriminalité ou au «broutage »}

Elles s'appréhendent à travers, d'une part les différents types de cybercriminels ou «brouteurs » et leurs modes opératoires dans lesquels interviennent les crimes rituels d'autre part.

\section{Différentes catégories de cybercriminels}

Les enquêtés ont pu identifier quatre (4) types de brouteurs sur la base des variables telles que l'âge, le niveau d'étude, le statut socioprofessionnel et le sexe : Les novices, les amateurs, les professionnels et les experts.

\section{$\checkmark \quad$ Les novices}

Les novices sont des jeunes de sexe masculin dont l'âge est compris entre 13 et 7 ans, pour la plupart, élèves du secondaire. Ils perçoivent les cybercafés comme des lieux de prédilection parce qu'ils y découvrent pour la première fois l'arnaque en ligne et s'y adonnent.

\section{$\checkmark \quad$ Les amateurs}

Les amateurs sont les personnes issues de la tranche d'âge 18-23 ans, qui ont un niveau d'étude secondaire. Ils ont une connaissance de l'outil informatique, mais cela ne fait pas d'eux des maitres de l'arnaque, car ils opèrent toujours sous la supervision de jeunes plus âgés qu'eux et/ou d'anciens dans le domaine.

\section{Les professionnels}

Les professionnels sont des personnes âgées de 24 à 29 ans, qui ont pour niveau d'étude le supérieur. Ils sont très organisés et souvent pros de 
l'informatique. Ils se font l'honneur de franchir des zones de sécurité des réseaux les plus protégés, méconnus de tous et que seuls les experts des NTIC connaissent (cracker).

\section{$\checkmark \quad$ Les experts}

Les experts sont les brouteurs issus de la tranche d'âge $30-34$ ans, appelés généralement "Hacker " c'est-à-dire les personnes passionnées d'informatique. Ces derniers, par plaisir, curiosité, pénètrent les systèmes informatiques sans l'aide de manuel technique attaquent puis détruisent les informations d'accès à un système informatique afin d'en tirer avantage personnel (par exemple fraude par carte de crédit...). Ce sont des jeunes sans emplois fixes qui à longueur de journée passent leur temps à échanger avec une connexion personnelle qu'ils ont installés chez eux.

\section{Modes opératoires des cybercriminels}

Dans le but d'extorquer de fortes sommes à leurs victimes, les cybercriminels se livrent à diverses arnaques en ligne. Aussi, ont-ils recours aux pratiques occultes, voire sataniques afin que les arnaques soit fructueuses. Mais comment les cybercriminels s'y prennent pour la commission des crimes rituels ? Quels sont leurs différents stratagèmes?

\section{$\checkmark \quad$ Par une fausse identité}

Cette méthode consiste à se faire passer sous un faux nom et utiliser des photos d'une tierce personne obtenue par piratage de son compte email et avoir un échange permanent avec d'autres internautes. Toujours dans le but d'arnaquer le cybercriminel fait appel aux féticheurs pour pouvoir toujours avoir le succès dans ces entreprises. Selon un propos recueillir dans un journal de la place un ex-cybercriminel nous relate son mode opératoire : BECAO la fortune "j'ai commencé à me fait passer pour jeune noire de 18 ans un cour à prendre pour les hommes de 40 ans et plus. Ainsi, je sollicitais les services des jeunes filles du quartier pour me faire passer pour celles avec qui les blancs causaient quand ils rentraient en contact avec moi. Puis une fois rassurés, ils m'entretenaient avec de l'argent soit 3,9 millions la première année. Ainsi les choses sont devenues compliquées quand la police s'est mise à nos trousses. Il fallait trouver un moyen d'échapper à la police vu le nouveau rythme de vie que j'avais; l'un de mes amis issus de notre milieu de cybercriminels m'a mis en contact avec un féticheur béninois. C'est lui qui m'a " préparé " il m'a dit qu'on devait trouver de la salive de cadavre et un bouc noir pour faire un rituel dans la nuit au cimetière. On s'est infiltrés aux cimetières et on a fait des incantations sur une tombe en fendant un animal vivant en deux. Nous avons entendu des pleurs et des voix dans le cimetière puis le féticheur m'a remis un canari que je devais porter en courant sans regarder en arrière jusqu'à chez moi, une fois arrivée j'ai regardé dans le canari il avait beaucoup de billets de 
banque environ 500 milles et ceci chaque jour que je devais dépenser toute la journée sinon j'aurais un malheur je ne devrais pas dormir les nuits

\section{$\checkmark \quad$ Par la vente d'objets en ligne}

Ces cybercriminels se font passer pour des commerçants sous le nom de sociétés fictives basées en COTE D'IVOIRE pour la vente d'articles, matériels, vêtements et bien d'autres à des exportateurs étrangers ou personnes intéressées via les réseaux sociaux. Ils mettent tout en œuvre afin d'installer une confiance entre eux et l'acheteur avec l'aide de leurs féticheurs qui les sert de guide. Celui-ci par ses pouvoirs mystiques envoûte les acheteurs qui par la suite font un transfert d'argent par des virements bancaires ou numéro de carte bancaire pour l'objet virtuel. Un propos recueillir d'un ex-cybercriminel durant nos enquêtes : «je m'appelle XCFA LA MERVEILLE, moi par contre je pratiquais le commerce en ligne d'objets virtuels, animaux sauvages... sur les réseaux sociaux, ensuite avec la collaboration de mon féticheur je trouvais beaucoup de victimes, mais en échange d'avoir des rapports avec des personnes folles ou des cadavres et je gagnais au moins plus de 1000 euros dans la semaine $\gg$.

\section{$\checkmark \quad$ Usurpation de compte mail}

Il fait de grandes escroqueries, via le compte mail de sa victime et sans celle-ci s'en plaigne. Tout cela avec l'aide du mystique. Un autre témoignage présenté par un bouteur: PANTHERE NOIRE «moi une fois entré dans le compte mail de ma victime je prends sa photo que j'obtiens de son compte et je la remets à mon féticheur qui l'envoute pour ne pas que celle-ci une fois au courant du problème de son compte mail ne me traduise devant les juridictions par enquête de la police, et ce, en échange d'organes humains pour des rituels. Avec l'aide d'un ami à moi nous avons capturé un enfant à qui nous avons enlevé la langue et le poignet que nous avait recommandés notre féticheur afin d'avoir beaucoup d'argent et d'éviter la prison ».

\section{Demandes d'aides}

Les cybercriminels utilisent des courriers de demandes d'aides très attrayantes dont parfois ONG pour des enfants démunis en Afrique ; aide aux enfants orphelins de guerres; soutien aux femmes battues ou excisées en Afrique... et pour que cette demande d'aide leur soient parvenus ils demandent les services d'un mystique.

Témoignage d'un cybercriminel nommé, MACHINE DES SOUS "Après avoir créé une ONG via le net, notre demande d'aide tardait et il nous fallait rendre les choses plus rapides puisque ceux-ci étaient un peu hésitants pour le transfert de fonds. Alors nous avons été voir un grand féticheur pour accélérer notre démarche, chose qu'il a faite sans problème en échange des parties de notre corps que nous lui avons donné sans 
réfléchir ; moi j'ai donné mes deux doigts en échange pour les rituels et mes deux amies ont remis à leurs tours leurs fertilités sexuelles".

\section{$\checkmark \quad$ Par tchat love}

Cette technique consiste donc à discuter sur des sites de rencontres via internet avec » le mougou" comme ils se plaisent à appeler les Européens. Les "gais «', le nom de code des cybercriminels (brouteurs), discute donc avec ces Européens pendant des heures, voire des semaines entières. Est directement et avec webcam d'où certaines jeunes filles amies aux cybercriminels se plaisent à jouer aux jeux de la séduction avec les Européens afin de les rassurés et soutirer un maximum d'argent. Tous pour la plupart s'adonnent à des pratiques mystiques telle que (relations sexuelles avec personnes du même sexe ; l'eau extraite de cadavre; ne pas tendre la main a une femme en période de menstrues... tout cela avant de s'asseoir devant l'ordinateur, témoignage révélé d'un cybercriminel.

\section{$\checkmark \quad$ La technique de l'héritage}

Dans cette technique, le cybercriminel se fait passer pour une personne très malade (cancer, tumeur, sida...) dont les parents sont décédés et souhaitent mettre son héritage au service d'orphelins et biens d'autres personnes dans le besoin. Toujours dans l'objectif d'escroquer les victimes, ils leur demandent de prouver leurs bonnes fois en faisant parvenir une certaine somme d'argent en vue de démontrer un dévouement pour les œuvres caritatives et leurs prises de possession de l'héritage légué par les parents vus n'y arriveront pas toutes par faute de santé. Un mail qu'ils font circuler à des milliers d'adresses électroniques chaque jour est appelé dans leurs jargons « le format». Une fois que les victimes ont mordu à l'hameçon, ils demandent aux victimes potentielles de fournir des documents (photocopie de passeport, les certificats dépôts).

Les cybercriminels font immédiatement appel aux pratiques rituelles afin de celle-ci fasse parvenir l'argent dans les jours qui suivent et obtenir d'avantage de nombreuses victimes au prix de sacrifices rituels.

\section{$\checkmark \quad$ La technique de la distribution}

Cette technique consiste donc à faire à une répartition des virus informatiques vers les ordinateurs des potentielles victimes. Ils mettent en place un mécanisme en vue d'atteindre un but. Il s'agit de l'envoi d'un grand nombre de courriers électroniques indésirables. Ces virus prennent possession de la messagerie de la victime et s'apprêtent à se déployer dans tout le système.

\section{$\checkmark \quad$ La technique du jeu de loterie par de faux documents}

Appelé «' format »' par les cybercriminels cette technique consiste donc a confectionné des documents administratifs qui portent le nom, des logos, des indications précises et des cachets de certains ministères bien ciblés. Ce stratagème mis en place par ceux-ci n'est autre que sur la 
supervision maîtresse du grand féticheur qui dans cette affaire envoûte les victimes afin que ceux-ci envoient davantage de l'argent,

\section{Les techniques utilisées par les criminels lors des crimes rituels}

— Enlèvement: Nos enquêtés nous ont fait part de l'enlèvement. Ils soutiennent que les individus sont enlevés par des individus dont on ne maitrise par la provenance. Cela se fait très souvent à des heures tardives. En ce qui concerne l'enlèvement des enfants, celui-ci a très souvent lieu au bord des établissements scolaires dans la journée.

- Mutilation: La mutilation est une atteinte volontaire à l'intégrité physique d'une personne entrainant la perte d'un membre ou d'un organe.

- La séquestration: La séquestration est 1'action de priver une personne de sa liberté en la maintenant enfermée. Les individus sont séquestrés par les ravisseurs et exécutés par la suite.

- La violence: Les bourreaux utilisent la violence pour maitriser leurs victimes. À cet effet Tonda (2014) soutient que : « ce ne sont pas des crimes rituels, mais de la barbarie ». Il veut montrer le caractère brutal des criminels. Girard (2011) parle de la violente mise à mort de la victime.

- Exécution: L'exécution est l'acte d'homicide qui consiste à tuer une personne.

- Prélèvement: Le prélèvement est l'action de prélever. Les organes des victimes sont prélevés.

\section{Facteurs explicatifs du phénomène}

En Côte d'Ivoire, plus particulièrement dans le milieu abidjanais, une partie de la jeunesse a choisi de faire des cybercafés un espace de prédilection. Certains dépassent ce stade, sacrifient des parties de leur corps afin de devenir millionnaires. D'autres «trouvent parfois la mort » dans ces histoires d'arnaques basées sur les crimes rituels. Malgré cela, le phénomène prolifère. Pourquoi de tels agissements ? Qu'est-ce qui peut bien motiver les auteurs de tels comportements ? Les facteurs explicatifs des crimes rituels rattachés au broutage sont tantôt d'ordre socio - économique, tantôt d'ordre humain, matériel et juridique.

\section{Facteurs d'ordre socio-économique}

\section{$\checkmark \quad$ L'obligation de s'enrichir davantage pour être toujours compétitif}

L'incapacité d'accès aux biens et matériaux de base (manque de soins ; de nourriture ; d'habitat et d'éducation), conduisent certains individus à développer des comportements délinquants.

$\mathrm{X}, 18$ ans (cybercriminel) affirme, «moi je suis élève en classe de seconde, mon papa travaille plus c'est dur à la maison si je ne fais pas de 
sacrifices pour envouter les gens sur le net là je ne peux même pas manger ni m'habiller comme mes amis, en plus je donne aussi à la maison c'est ça on prend pour faire nos dépenses, et personne ne me demande d'où je sors cet argent $\gg$.

X, 24 ans (cybercriminel) affirme ceci : « moi mes parents $n$ 'ont rien, en plus je ne travaille pas, je suis étudiant je suis conscient que ce n'est pas bien, mais c la pauvreté qui nous oblige, je vais sur internet pour gagner mon jeton un peu un peu avec l'aide de mon féticheur pour ne pas qu'on $m$ 'attrape et me jeter en prison. "Et puis il n'y a rien de mal c'est le mouvement après tout".

G, 42 ans (homme mystique) : "tout est chère, la vie est devenue très difficile, et ya pas l'argent les jeunes-là ne travaillent pas, s'ils vont sur le net pour avoir un peu d'argent dans la main des blancs ou autres. Où est le mal dans ça? en plus s'ils demandent mon aide pour devenir riche, je le ferais".

$\mathrm{X}, 17$ ans (cybercriminel) affirme : "le mouvement coupé décalé fait fort a babi si tu n'a pas les moyens tu ne peux pas fait partir des grands milieux de show night-clubs de la nuit. Moi mes parents n'ont rien, je suis élève et je veux fait comme les Douk saga donc j'ai eu mon petit coin sur le net pour Bara " travail"' avec l'aide d'un féticheur aujourd'hui je suis un dur dans la cour des grands de la nuit".

\section{$\checkmark \quad$ La cherté de la vie}

Le coût trop élevé de la vie, c'est-à-dire la flambée des prix des denrées alimentaires, vestimentaires, le transport et de l'habitat rend la vie très difficile puisque les personnes sont incapables de se procurer les produits pour survivre.

\section{$\checkmark \quad$ Le manque d'emploi}

Une partie de la population s'est passionnée vers les crimes rituels via l'outil informatique pour combler le vide laissé par le désespoir et la recherche d'un avenir meilleur. Selon l'AGEPE, le taux du chômage est de plus de $45 \%$ pour la population active (population diplômée).

\section{$\checkmark \quad$ Les crimes rituels liés au broutage : une activité rentable}

Le secteur des crimes rituels dans la cybercriminalité est une véritable entreprise qui rapporte d'énormes bénéfices à ses auteurs qui peuvent obtenir près de trois milles Euro (3000 €) soit 198650000 FCFA de son correspondant via le net.

\section{$\checkmark \quad$ L'influence des médias sur la jeunesse}

Les médias constituent un facteur aussi important dans l'évolution de ce phénomène en ce sens que la consommation devient un but à atteindre et provoque un renversement de valeurs. Par exemple la publicité crée des besoins artificiels chez les consommateurs et les emmène à envisager certains objets de luxe comme des biens essentiels. 


\section{Imitation des précurseurs du mouvement "coupé décalé »}

Un rythme urbain crée par des jeunes ivoiriens vivant à l'étranger qui arnaquaient avec des stratagèmes bien huilés des Européens via les réseaux sociaux et qui par la suite se sont fait remarqués très bien habillés, coiffés en distribuant de l'argent pendant leurs prestations scéniques et les shows en Côte d'Ivoire. Ainsi ils avaient créé le mouvement du "' coupé décalé »' dans lequel le phénomène du "travaillement " et du " boucan". Très tôt certains jeunes ivoiriens se mettront eux aussi à vouloir se faire de l'argent facile avec le même procédé que leurs frères vivants à l'étranger.

\section{Facteurs humains et matériels}

\section{$\checkmark \quad$ Corruption des agents de transfert d'argent}

Reconnu pour être rapide et fiable, ces agences de sécurité présentent des failles, car la plupart des cybercriminels bénéficient pour leurs activités, de la couverture de certains agents avec lesquels ils partagent le pourcentage du gain dû à l'arnaque

\section{$\checkmark \quad$ Collaboration force de l'ordre - cybercriminels}

Cette collaboration se traduit donc par le fait que les cybercriminels au cours de leurs manœuvres criminelles lorsqu'ils sont pris par certaines forces de sécurités, sont relâchés parce qu'ils échangent leurs libertés aux prix de nombreux billets de banque d'où les crimes restent non déclarés dans les rapports de postes de sécurités.

\section{$\checkmark \quad$ Insuffisance de personnels de lutte contre phénomène}

$\mathrm{Au}$ cours de nos recherches sur terrain, nous avons pu observer l'insuffisance d'agents de lutte contre ce phénomène, et les difficultés rencontrées dans l'exercice de leurs missions à savoir la non-collaboration des banques ou des agences de transferts de fonds qui crée une difficulté dans l'accès aux informations concernant l'identité du cybercriminel.

\section{$\checkmark \quad$ Insuffisance de matériels adéquats}

La démocratisation et la vulgarisation des NTIC dans nos sociétés ont favorisé l'accès à internet pour tout l'ensemble de la population, surtout qu'un ministère (NTIC) a été créé en vue de faire la promotion du secteur des télécommunications en Côte d'Ivoire. Cependant, nos enquêtes auprès des agents que nous avons interrogés, montrent qu'ils rencontrent de grandes difficultés dans l'exercice de leurs missions à savoir : peu de véhicules pour l'exercice de leurs patrouilles; et une insuffisance au niveau de matériels informatiques qui permettront de tracer à distance les cybercriminels dans leurs manœuvres criminelles. 


\section{Facteurs d'ordre juridique}

L'absence de cadre juridique adéquat favorise l'évolution des crimes rituels dans la cybercriminalité en ce sens que conscient que le droit ivoirien ne prévoit de sanction contre ces agissements. La cybercriminalité continue d'être pratiquée et même prend de nouvelles formes d'où les crimes rituels associés. Au terme de notre analyse concernant les facteurs explicatifs des crimes rituels dans la cybercriminalité, nous pouvons dire que l'apparition des NTIC en Côte d'Ivoire, bien qu'elle eut participé au développement, a suscité un espoir et un enthousiasme chez des individus et a fait naitre une nouvelle forme de criminalité : les crimes rituels liés à la cybercriminalité

\section{Conséquences}

L'accessibilité à internet pour tout l'ensemble de la population laisse entrevoir aujourd'hui de nombreux crimes rituels commis via les réseaux sociaux. La jeunesse ivoirienne s'adonne de plus en a ce phénomène qui prend davantage de l'ampleur. La fréquence des crimes rituels liés à la cybercriminalité, ternit davantage l'image de la Côte d'Ivoire en ce sens que les médias étrangers réalisent des reportages sur la cybercriminalité et les crimes qui s'en suivent.

\section{Problème sécuritaire sur le plan local et international}

Indiquer désormais comme l'un des pays à « haut risque d'insécurité », l'économie ivoirienne risque gros par l'expansion de ce phénomène du point de vue international.

$\mathrm{Au}$ cours de notre enquête, nous avons observé que le phénomène influence négativement les jeunes à telle enseigne que suite aux pratiques fétichistes qu'ils opèrent pendant leurs arnaques via les réseaux sociaux, on constate un chiffre croissant de personnes sans vie; des organes humains retirés en pièces détachées ; des bébés et enfants tués...

Un tableau est donc construit selon l'évolution de ce phénomène au fil des temps avec un échantillon de 725 personnes.

Tableau 2. Évolution des pertes en vies humaines dues aux crimes rituels liés a la Cybercriminalité par quinquennat

\begin{tabular}{|c|c|c|c|c|c|c|}
\hline Année & $\begin{array}{c}1990- \\
1995\end{array}$ & $\begin{array}{c}1996- \\
2000\end{array}$ & $\begin{array}{c}2001- \\
2005\end{array}$ & $\begin{array}{c}2006- \\
2010\end{array}$ & $2011-2015$ & TOTAL \\
\hline $\begin{array}{c}\text { Nombre de } \\
\text { victimes }\end{array}$ & 15 & 62 & 93 & 203 & 352 & 725 \\
\hline Pourcentage $\%$ & $2,06 \%$ & $8,55 \%$ & $10,06 \%$ & $28 \%$ & $48,55 \%$ & 100 \\
\hline
\end{tabular}

Source : nos enquêtes

D’après nos observations mentionnées dans le tableau ci — dessus, nous pouvons dire que de 1990-1995 on avait un taux de 2,06 \% ; de 19962000 un taux de 8,55\%; de 2001-2005 un taux de 10,06 \% ; de 2006-2010 
un taux de $28 \%$; et enfin de 2011-2015 un taux de 48,55\%. Ceci explique que la cybercriminalité existait depuis bien longtemps et n'avait pas pris cette nouvelle forme à savoir les crimes rituels.

\section{Types de crimes rituels/pertes en vies humaines}

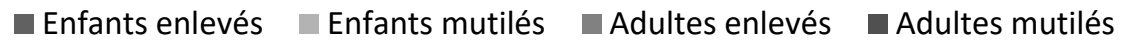

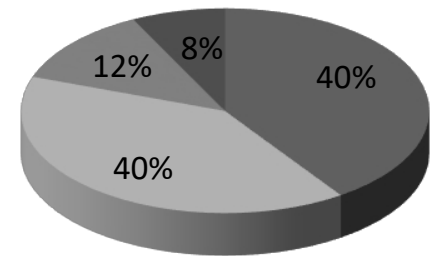

Figure 1. Types de crimes rituels/Pertes en vies humaines

Par cette figure ci-dessus nous pouvons expliquer que, la plupart des personnes enlevées et mutilées à Abidjan sont commises dans la plus grande commune qu'est Yopougon. Ainsi, on observe un taux de 40,41 \% d'enfants enlevés et $40 \%$ d'enfants mutilés, ensuite un taux de $12,13 \%$ d'adultes enlevés et 7,44\% adultes mutilés.

Ainsi nous remarquons que, les enfants sont les plus touchés par de phénomène, car ils sont les plus vulnérables et sont considérés comme des personnes pures et saines pour toute réussite dans la pratique rituelle ou du « zamou ».

\section{Les crimes rituels liés à la cybercriminalité: une plaie pour le Système éducatif}

Les jeunes abandonnent les études et préfèrent passer plus de temps sur les réseaux sociaux et les endroits pour des pratiques fétichistes et cyberespaces qui leur procurent l'argent rapide, chose que l'école ne peut offrir dans l'instant. La découverte de cette nouvelle quête du gain rapide conduit les jeunes à se détourner des valeurs telles que : le goût à l'effort ; le respect... cependant ce tableau ci-dessous nous permettra d'observer l'utilisation passionnée qu'ont ces jeunes pour les réseaux sociaux informatiques au fils des années. 
Figure 2. Répartition du nombre d'internautes par année

Source: ATCI

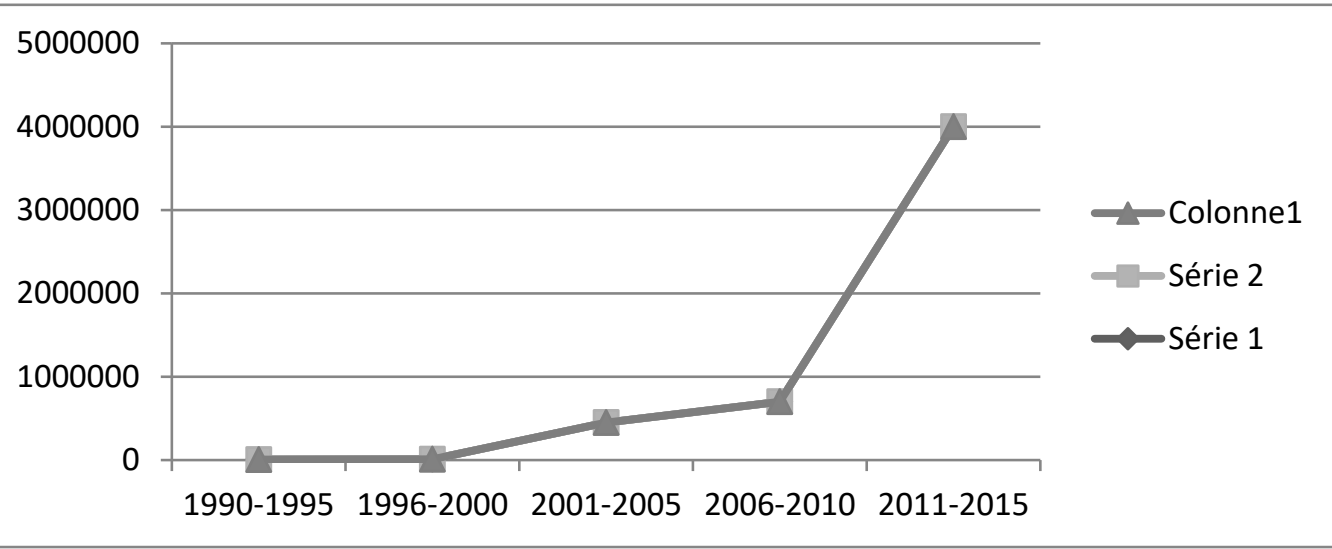

Selon la courbe dressée ci-dessus, le phénomène évolue au fil des temps, car on constate que, plus le temps passe, plus la jeunesse s'y intéresse davantage au détriment des études.

On constate que de 1990-2000, la courbe la courbe a légèrement monté de 600010000 internautes et de 2001-2010 on observe une évolution rapide de 450000-700000 internautes et 2011-2015 on remarque 4000000 internautes.

Le goût au gain facile est devenu aujourd'hui la préoccupation majeure de la plupart des jeunes ivoiriens qui ne voient qu'en ce phénomène un « business » très prometteur.

La commune ivoirienne est marquée par le culte de la médiocrité, l'absence d'exemplarité, de jeunes élèves de lycées et collèges, étudiant qui reçoit de mauvais signaux et l'école devient une antichambre pour ceux-ci.

\section{Conséquences au niveau culturel}

Le fétiche est une culture africaine, une pratique utilisée couramment pour des guérisons diverses. En Côte d'Ivoire, le fétiche est utilisé le plus souvent pour la confection d'objet sacré par exemple des masques, ponts ou autres sculptures qui autrefois étaient une fierté ivoirienne parce qu'ils invitaient au tourisme. Aujourd'hui, il est utilisé par des jeunes ivoiriens à des fins criminelles qui bouleversent même des tabous ivoiriens, ce qui pourrait à la longue détruire l'éthique et la morale de la société.

\section{Conséquences au niveau économique}

L'expansion des crimes rituels associés à la cybercriminalité touche la réputation des banques qui servent de façon indirecte aux retraits du gain de l'arnaque avec la collaboration indécente de certains agents de la banque. Parfois certains clients de banques ont peur d'utiliser les services en ligne que proposent les institutions financières ivoiriennes. 
Cette méfiance s'observe par le fait que les clients ne maitrisent pas bien le moyen technique pour authentifier les sites de consultation de crédit en ligne et craignent de se tromper pour laisser grandement ouvertes leurs coordonnées bancaires à la portée de ces passionnés informatiques (cybercriminels). Alors un doute sur la fiabilité des banques s'installe et constitue un danger pour les institutions financières. L'ampleur que prend ce phénomène devient très inquiétante, car de nouvelles techniques et méthodes prennent forme. En effet, il est difficile pour les opérateurs économiques locaux ou internationaux d'effectuer des transactions via le Net ou en ligne à partir de la Côte d'Ivoire. La mauvaise réputation qu'a la Côte d'Ivoire en matière de cybercriminalité s'est de plus en plus accentuée depuis l'avènement de la nouvelle forme à savoir: les crimes rituels liés à la cybercriminalité, qui constitue un problème très important dans l'économie du pays en ce sens qu'elle occasionne la fuite de nombreux investisseurs étrangers vers d'autres pays jugés plus sécurisés. L'innovation dans le domaine économique (inter bancarisation) et les échanges d'information via le net (conférences, les cours en lignes internationales vers la Côte d'Ivoire) risque de compromettre les relations internationales ivoiriennes, car le pays sera mis sur le banc des accusés d'où un risque d'être isolé.

\section{Le sentiment d'insécurité}

Tableau 3. Répartition des réponses données par les enquêtés à propos de l'existence d'un sentiment d'insécurité dans les communes de Yopougon et d'Abobo.

\begin{tabular}{|c|c|}
\hline Existence d'un sentiment d'insécurité & Pourcentage \\
\hline Oui & $98,03 \%$ \\
\hline Non & $01,96 \%$ \\
\hline Total & $100 \%$ \\
\hline
\end{tabular}

Source: Enquêtes

Les enquêtés estiment en majorité avec $98,03 \%$ qu'il existe un sentiment d'insécurité à Yopougon et Abobo contrairement à 1,96\% qui rejettent cette thèse.

Tableau 4. Le degré d'insécurité au sein de la commune d'Abobo

\begin{tabular}{|c|c|}
\hline $\begin{array}{c}\text { La situation sécuritaire à Yopougon et à } \\
\text { Abobo }\end{array}$ & Pourcentage \\
\hline Très peu sure & $74,50 \%$ \\
\hline Assez sure & $17,64 \%$ \\
\hline Très sure & $7,84 \%$ \\
\hline Total & $100 \%$ \\
\hline
\end{tabular}

Source: Enquêtes 


\section{Les meurtres}

Selon l'article 342 du Code pénal ivoirien, est qualifié de meurtre l'homicide commis volontairement.

Tableau 5. L'existence de meurtres au sein de la population de Yopougon et d'Abobo

\begin{tabular}{|c|c|}
\hline Existence de meurtre & Pourcentage \\
\hline Oui & $100 \%$ \\
\hline Non & $00 \%$ \\
\hline Total & $100 \%$ \\
\hline
\end{tabular}

Source: Enquêtes

Les statistiques du tableau 14 traduisent le fait que tous les enquêtés sont unanimes sur le fait qu'il existe des meurtres au sein de la population de Yopougon et d'Abobo.

\section{Mesures en vigueur et politiques de lutte envisageables}

Depuis un certain moment, le phénomène "' du broutage $3 \mathrm{G}$ »' ou crime rituel associé à la cybercriminalité a amené des cybercriminels à tuer d'autres hommes dans le but de se faire de l'argent via le net. Ainsi vu la proportion démesurée de ces actes délinquants dans la commune de Yopougon, les autorités se sont vues dans l'urgence de mettre en places des stratégies de lutte contre ce phénomène.

Au nombre de ces actions mises en place, nous avons deux catégories de luttes :

- $\quad$ Catégorie théorique (la mise en place des assises numériques; la création d'une loi...).

- $\quad$ Catégorie pratique (création d'organes spécifiques de lutte).

\section{Mesures en vigueur}

\section{Aspects théoriques : mise en place des assises du numérique}

Lors des assises du numérique en Côte d'Ivoire, il était question de débattre sur la problématique liée à l'évolution du numérique avec pour thème central cyberespace et données personnelles. Au cours de ce forum il était question d'attirer le secteur formel sur la nouvelle forme de criminalité via les réseaux sociaux puis l'importance d'un contrôle de sécurité en ligne. Acteur majeur du secteur des NTIC, Microsoft Afrique de l'Ouest et du centre, à travers des conférences a permis de tracer les voies pour un début de sécurisation du cyberespace ivoirien d'où la création d'organes spécifiques de lutte contre les crimes rituels cybercriminalité. Le principal outil théorique de lutte contre la cybercriminalité en Côte d'Ivoire est la création d'une loi relative à la lutte contre ce phénomène. Le dispositif mis en place par cette loi est essentiellement répressive, crée des nouvelles infractions pénales et adapte des infractions classiques aux NTIC. L'accès 
frauduleux à un système d'informations, l'introduction frauduleuse de données dans un système d'informations, l'altération, la modification ou la suppression frauduleuse de données informatiques, l'utilisation frauduleuse d'éléments d'identification d'une personne physique ou morale par le biais d'un système d'informations, l'envoi de messages électroniques non sollicités (spam), la suppression ou le détournement de correspondances électroniques. L'usurpation d'identité est prévue et réprimée à l'article19 selon lequel, «est puni de deux à cinq d'emprisonnement et de 5000000 [7500euros] à 10000000[1500 euros] de francs FCFA, quiconque utilise frauduleusement un ou plusieurs éléments d'identification d'une personne physique par le biais d'un système d'information ». L'utilisation, la possession, l'offre, la vente, la mise à disposition, la transmission « en toute connaissance de cause de fausses données d'identification d'une personne physique ou morale par le biais d'un système d'information » sont également réprimées.

\section{Aspects pratiques : organes et actions de lutte contre le phénomène \\ $\checkmark \quad$ La création d'une police scientifique}

Conformément aux articles $\mathrm{n}^{\circ} 41$ à 44 dudit décret depuis sa création 08 mai 2007 par le décret $n^{\circ} 2007-464$ sous organisation du ministère de l'Intérieur et de la direction générale adjointe chargé de la police scientifique contiennent, un (1) cabinet et trois (3) directions à savoir :

La DITT qui est un service de police qui ne traite que la cybercriminalité dans laquelle sa mission est :

- La recherche et la documentation sur les nouveaux types de criminalité et nouvelles technologies ;

- $\quad$ D'examiner des documents d'études d'écritures manuscrites et de la dactylographie, des révélations et des études de traces ;

- De la mise en place d'outils informatiques, de techniques et de moyens scientifiques nouveaux en matière de lutte contre la criminalité ;

- Du traitement des signaux audio, de l'image et de l'expertise informatique.

Elle est composée de deux (2) sous-directions dont :

- $\quad$ la sous-direction des traces technologiques (SDITT) ; en accord avec le ministère ivoirien de l'Intérieur et l'agence de télécommunications de Côte d'Ivoire (ATCI), la plateforme de lutte contre la cybercriminalité a été créée en 2011 dont l'objectif est la création de coopération de lutte contre la cybercriminalité.

La PLCC, se compose d'enquêteurs, ingénieurs et techniciens de la DITT, la direction de la police économique et financière (DPEF) et l'autorité de régulation des télécommunications de Côte d'Ivoire (ARTCI). La PLCC a mis en place un système de plainte en ligne. Elle dispose de conseils et avis 
de recherches d'escrocs identifiés en utilisant un moteur de recherches de vérification de numéros de téléphone, un e-mail, un pseudo impliqué dans une affaire de ce phénomène. Sa mission première est d'effectuer des enquêtes judiciaires sur les infractions visant ou utilisant des systèmes informatiques, et portant également sur les modes de traitement, de stockage et de transmission de l'information ; Apporter son assistance technique aux services de police et aux services connexes chargés de l'application de la loi lors des enquêtes judiciaires; Contribuer à la mise en place de moyens techniques et au développement de l'expertise pour l'examen et le traçage des systèmes d'information, et notamment l'audit et l'autopsie des disques durs d'ordinateurs, des téléphones et d'autres médias de stockage et de traitements de l'information.

\section{$\checkmark \quad$ La coopération internationale}

Un atelier d'échange stratégique sur la nouvelle forme de crime via les réseaux sociaux initiés par les réseaux FRANCOPOL et les autorités de la police nationale de Côte d'Ivoire. Et une convention a été signé entre la direction de l'informatique et les traces technologiques (DITT) et le groupe IB MAROC, dans un cadre de partage d'expérience et un appui stratégique dans le domaine des technologiques et de l'information. La sensibilisation est très importante dans la lutte contre la cybercriminalité, l'État à sensibiliser la population de façon originale c'est-à-dire que la sensibilisation s'est faite à travers des films, et séries, télévisées. En effet le message diffusé par ces séries est que : "l'internet est un outil de communication et non d'arnaque." La création de cette école est très nécessaire en ce sens que le pays manque de personnes qualifiées dans le domaine des NTIC, afin de trouver de bonnes stratégies de lutte contre le phénomène via le Net. L'ampleur que prend ce phénomène aujourd'hui a favorisé la mise en place de structures, d'organisation non gouvernementale en partenariat avec le ministère de la Famille, de la femme et de l'enfant dans le but de lutter contre la progression de ce phénomène. Nous avons MVCI (mouvement pour la promotion des valeurs nouvelles en Côte d'Ivoire); GOFEHF (groupe d'organisation féminine); ONG protégez nos enfants ; ONG N'assassinez pas nos enfants.. , tous ont mis en place de façon urgente et systématique un comité de crise au plan national ainsi que des sous-comités dans les communes d'Abidjan et à l'intérieur du pays et l'interdiction systématique et formelle de véhicules a vitre opaque et plaques banalisées.

\section{Propositions de mesures}

La Côte d'Ivoire aujourd'hui souffre d'un grand mal surtout quand on reçoit des images insoutenables d'enfants, adultes atrocement mutilés, par des criminels obscurantistes (cybercriminels ou brouteurs, marabouts). La responsabilité de l'État est donc grande. 
Ainsi dans le but d'aider l'État ivoirien dans sa lutte perpétuelle contre ce phénomène opéré par les jeunes brouteurs nous essayons de soumettre quelques propositions de mesures qui a notre avis pourrait apporter un plus. Nos propositions se situent à deux (2) grands niveaux :

- Le niveau répressif.

- Le niveau préventif.

Niveau répressif

\section{$\checkmark \quad$ Mise en place de cadre juridique}

La répression de tout acte demande au préalable deux (2) conditions réunis à savoir : l'existence d'un acte criminel et une loi qui l'interdit. Pour cela, il faudrait pour nous que le législateur ivoirien en se servant des textes juridiques étrangers s'adapte aux normes du Code pénal ivoirien pour édicter des textes juridiques. Ainsi pour notre part, les auteurs des crimes rituels opérés dans la cybercriminalité doivent encourir de peine grave allant cde 10-20 ans de prison ferme avec une amende de 1000000 à 700 millions $\mathrm{F}$ CFA. La mise en place d'une plate-forme d'échange nationale permettra d'effectuer une collaboration plus sérieuse avec les établissements numériques et les services de forces de l'ordre et de sécurité sur le traçage à distance du caractère illicite qu'utilise le cybercriminel dans son opération. Au cours de nos recherches, nous avons constaté l'insuffisance d'agents de lutte d'une part et un sous équipement en matériel d'autre part. Il sera donc raisonnable pour le gouvernement ivoirien d'améliorer ce déficit en mettant à la disposition des agents formés dans la lutte contre ce phénomène des moyens de logistiques pour une intervention rapide lorsqu'un cas est signalé dans une zone. La mise en place d'un cadre répressif nous sera utile en ce sens que le recensement des hommes mystiques permettra la réglementation et le contrôle permanent de leurs activités.

\section{Niveau préventif}

\section{$\checkmark \quad$ Au niveau des populations}

À ce niveau, une collaboration population-force de l'ordre; une campagne de sensibilisation sur le respect de la valeur sacrée l'homme et sur les dangers des crimes rituels et de la cybercriminalité serait efficace. La collaboration population force de l'ordre est d'une importance capitale, car, elle se manifestera par la dénonciation de tout type d'acte criminel observé dans les communes ivoiriennes par la population aux postes de police. Il faudrait une campagne de sensibilisation auprès de la population sur le respect de la valeur humaine pour dire » qu'on ne devient pas riche par le sang humain, mais par le travail ». Le phénomène de crimes rituels et de la cybercriminalité est une infraction nouvelle qui a amené des jeunes ivoiriens avides de richesse à tuer des hommes à travers des pratiques occultes et euxmêmes terminé par une mort tragique. Concernant ce niveau, il faudra une 
collaboration des entreprises du secteur privé et du public; et un renforcement de coopération internationale. Les responsables du secteur privé et public doivent investir dans les programmes de recherches et de développement afin de rendre les systèmes plus sûrs. Au vu de nombreuses conséquences causées par les cybercriminels via les réseaux sociaux, le renforcement de la collaboration internationale sera d'une grande utilité dans la lutte contre ce phénomène qui mine davantage la société ivoirienne.

\section{Discussion et Conclusion}

Pour conclure nos recherches sur les crimes rituels liés à la cybercriminalité, nous pensons qu'il serait important de rappeler que ce phénomène est originaire du Nigeria acteur premier de la cybercriminalité en Afrique de l'Ouest. La cybercriminalité est arrivée dans les années 90, mais s'est révélée de façon générale en Côte d'Ivoire en 2002 pendant la période de crise politique qu'a connu notre pays et l'avènement du mouvement «' coupé décalé »' né de cette même période de crise, un rythme urbain créé par les jeunes ivoiriens vivant à l'étranger qui recommande la possession d'une sacrée somme d'argent pour être un des leurs. Vue le bruit qu'ils faisaient a chaque déplacement dans les endroits luxueux et night-clubs d'Abidjan par des distributions de billets de banque. Le concept du « travaillement » et du " boucan "prit forme. Un bon nombre de jeunes ivoiriens se sont vus imiter leurs aînés du mouvement en s'adonnant a des pratiques immorales à travers le net, d'où la naissance des crimes rituels dans la cybercriminalité.

Ainsi on observe beaucoup la pratique de ce phénomène chez des jeunes de 13-17ans de profession élève pour la plupart, 18-23ans de profession étudiants et enfin 24-34ans généralement des sans-emploi.

Les jeunes ivoiriens ayant désormais pris un goût au jeu de l'arnaque via le net, ont très vite poussés le bouchon plus loin ils usent de stratagèmes bien huilés pour escroquer leurs victimes via les réseaux sociaux en faisant intervenir les pratiques mystiques dans leurs arnaques. Ils abandonnent les bancs de l'école pour passer la plupart du temps dans les cyberespaces. Ainsi à travers des modes opératoires diverses (par la vente d'objet en ligne, usurpation du compte mail, faux document administratif...), les cybercriminels (novices, amateurs, professionnels et experts) passent a l'acte.

Ils fréquentent marabouts, féticheurs, devins, prêtres animistes... pour attacher leurs correspondants afin de vider leurs comptes bancaires sans que ces derniers s'en rendent compte. Cet effet magique est la clé de voûte pour faire couler des millions. De là suivent les recommandations fermes du grand sorcier prêtre à savoir les crimes rituels. Ce qui explique la croissance des personnes enlevées et mutilées dans la commune ivoirienne. 
Cependant, nous observons avec un échantillon de 725 personnes tuées ou enlevées un constat de $10,06 \%$ de pertes en vie humaines dans les années $2001-2005$; $28 \%$ en $2006-2010$ et $48,55 \%$ en $2011-2015$ avec $40,41 \%$ d'enfants enlevés et $40 \%$ d'enfants mutilés ensuite $12,13 \%$ d'adultes enlevés et 7,44\% d'adultes mutilés.

À travers ces données ci-dessus, on observe que les plus touchés par les pratiques mystiques sont les enfants à cause de leurs vulnérabilités.

Pendant nos recherches sur terrain nous avons pu remarquer que certains facteurs expliquent la persistance de ce phénomène dans la commune ivoirienne et se situe a trois (3) niveaux; le niveau socioéconomique (la pauvreté, la cherté de la vie, imitation des précurseurs du mouvement, la complicité de certains agents de police, gendarmes, administrateurs, agents de banques...), le niveau matériel et humain (manque de matériels adéquats, insuffisance d'agents de lutte contre ce phénomène...) et le juridique (manque de cadre juridique adéquat).

Cette jeunesse devenue partisane du moindre effort à cause du gain facile suite aux crimes rituels qu'elle opère dans la cybercriminalité pourrait engendrer de nombreuses conséquences à l'État ivoirien à savoir (une image négative du pays, problème sécuritaire sur le plan local et international, des pertes en vies...), au niveau culturel et économique.

La commune ivoirienne secouée par le phénomène tristement célèbre des jeunes cybercriminels s'est vue dans l'urgence de mettre en place des stratégies et moyens de lutte pour pouvoir contrer l'évolution de ce phénomène d'où la mise en place de forums, ateliers de formations, et la mise en place d'une police scientifique (DITT) et d'une plateforme de lutte contre ce phénomène (PLCC).

Tous les efforts consentis par l'État ivoirien paraissent insuffisants puisque le phénomène continue d'évoluer. Une raison pour nous d'apporter une aide dans la course à la lutte contre ce phénomène, c'est pourquoi nous avons proposé quelques stratégies sur deux (2) aspects : l'aspect répressif et préventif.

\section{References:}

1. Amilton, F. (2009). Le secret des jeunes riches, Magazine top visage, sur http// www.ivoirenews.ivoire-blog.com, consulté le 18 décembre 2009, à 16 h.

2. Bamba, L. \& Bazare, N. R. (2016). Pratiques festives des "' Brouteurs " ' en CI : entre ritualisation de la fête et catégorisation sociale des cybercriminels.

3. Bekanty, F. (2009). Cybercriminalité comment ils volent, escroquent et tuent sur internet?, sur http// www.newsabidjan. net/artticlen ๑3099455, consulté le 10 Octobre 2009 à 20 h 
4. Bogui, J.J. (2010). La cybercriminalité, menace pour le développement. Afrique contemporaine, Université du Québec, Montréal : PUM.

5. Essono, Tsimi, E \& Rifoe, P. PH. (2015). Afrique centrale : l'intégration par les « crimes rituels »? Pour l'autre afrik/mis à jour à 16 h 14 - Paris, www. Afrik.com/ariq-centrale-intégration-par- les crimes.

6. Goore-BI, M. (2008). Arnaque par Internet: la «mafia » de la cybercriminalité en Côte d'Ivoire. Qui sont-ils ? Comment opèrentils ? Quotidien 1'Inter du jeudi 15 mai 2008 (Abidjan).

7. Jacob, M. (2009). Voyage au cœur du cyber-crime avec Este. Sur http// www.globalsecuritymag.fr. Consulté le 04 Octobre à $10 \mathrm{~h} 11$.

8. Jeune Afrique (2015). Que sait-on des enlèvements des enfants ?

9. Kasse, R. (2009). Abidjan : les brouteurs sévissent. Blog personnel, sur http// www.regerkasse.pooire-blog.com, Consulté le 22 Septembre à $21 \mathrm{~h}$.

10. Kouassi, H. (2010). Economie numérique, 11éme édition des NTIC Les opérateurs économiques plaident pour un nouveau code des télécommunications, L'intelligent d'Abidjan n 1997 du mercredi 02 juin 2010.

11. Monnet, R. \& Franchin, F. (2005). Le business de la cybercriminalité, Hermès-Lavoisier.

12. Ouattara, L. (2010). Cybercriminalité en Côte d'Ivoire : 10 milliards de FCFA escroqués, policiers, gendarmes et procureurs impliqués. Quotidien 1'Expression $\mathrm{n}^{\circ} 290$.

13. Pilo, J. (2009). Gestion de communauté et de protection de la jeunesse face à 1'Internet,_sur http//www.info-utile.fr, consulté le 24 Septembre à $15 \mathrm{~h}$.

14. Rojinsky, C. (2000). Cyberespace et nouvelle régulation technologique, Dalloz.

15. Serres, D. \& Cluzeau (2008). La cybercriminalité : les nouveaux enjeux de la protection des données, Mémoire de Maîtrise, Faculté de droit, Université Laval du Québec.

16. Soudan, F. (2014). Afrique/Crimes rituels. Jeune Afrique.

17. Stamm (1994). Le sacrifice humain.SERGENT, (2011). Le sacrifice des femmes Samnites, pp 229-291.

18. Varenne, F. (2009). Qu'est-ce que l'informatique ? Edition Vrin. 